\title{
A Simple and Economical Approach/Concept to Evaluate Quality of Pharmaceutical Products Based on an Improved Dissolution Testing Methodology
}

\author{
Saeed A. Qureshi* \\ Therapeutic Products Directorate, Health Products and Food Branch (A/L 2202C1), Health Canada, Ottawa, K1A 0L2, \\ Canada
}

\begin{abstract}
Often the quality of drug products is evaluated based on chemical tests, commonly described in different pharmacopeias such as the USP. These tests includes: assay (potency), uniformity of dosage form and dissolution test. Presently, these tests are conducted separately with three procedures. Furthermore, dissolution tests are also conducted using multiple product-specific procedures. This makes the current practice of evaluation of the quality of pharmaceutical products complex and resource (human and financial) intensive. Recently a new improved dissolution methodology, based on a modified spindle known as crescent-shaped spindle, has been proposed. Using the proposed methodology, all these tests may be conducted based on a single dissolution test procedure. Such an approach would, therefore, provide a simpler alternative to the current practice with significant economical benefits. The attributes of the proposed approach which lead to the concept of product evaluation based on a single dissolution test procedure, are described here by testing a number of diltiazem products having different strengths and release characteristics.
\end{abstract}

The quality of drug products is evaluated based on chemical tests commonly described in different pharmacopeias such as USP. In general tests are conducted to establish presence of the expected amount of drug in the product (potency), uniformity or consistency of drug content in a product, such as, from tablet to tablet, and expected drug dissolution or release characteristics of a product. At present, these tests are conducted separately with at least three procedures. A new spindle known as crescent-shaped spindle has been proposed for improved drug dissolution testing [1-3]. The superiority of the new spindle appears to be due to its efficient extraction ability, because of improved productmedium interaction in the dissolution vessel. It may be argued that as all the above mentioned tests are based on the extraction of drug from a product, the new approach based on the new spindle may provide a common approach for these tests. This would provide a simpler alternative to the current practice, with a significant economical benefit.

This article provides a discussion and experimental evidence showing that a simpler and more relevant testing approach based on dissolution testing may be used for an overall evaluation of the quality of pharmaceutical products.

\section{MATERIALS AND METHODS}

Pharmaceutical Products: IR (30- and 60-mg) tablet and ER (120-mg once (CD) and twice (SR) a day) capsules of diltiazem products tested in the study were obtained from the local Canadian market. In total, 10 products were tested reflecting, 3 for each IR strengths, 3 for ER once-a-day and 1 for SR, from 3 manufacturers coded as manufacturers "A", "B" and "C".

*Address correspondence to this author at the Banting Research Centre $(\mathrm{A} / \mathrm{L}$ 2202C1), Tunney's Pasture, Ottawa, Ontario, K1A 0L2, Canada; Tel: 613957-3728; Fax: 613-941-8932; E-mail: saeedVंqureshi@hc-sc.gc.ca
All other chemicals and solvents were of analytical grade and used as supplied by the suppliers.

Stirring Spindle: The crescent-shaped [1] spindles were used for testing. The crescent-shaped spindle is designed to fit the commonly used apparatuses as a substitute for the Paddle or Basket spindle. The agitator has a stem part and the lower part is curved to conform to the shape of the vessel in which it is rotated, but with no direct contact with the surface of the vessel.

The end of the stem conforming to the bottom part of the vessel has filamentary elements filling the gap between the stem and the bottom part of the vessel. Therefore, when the device attached to the vertical shaft rotates, the brush-type agitator will sweep along the bottom and sides of the vessel providing even distribution and mixing of the disintegrating material thus avoiding accumulation (coning).

Instrumentation: The dissolution tests were conducted using a DISTEK 2100C system which is comprised of a bath with six vessels and met the physical and mechanical specifications as noted in the USP [4]. The dissolution tests were conducted using the crescent-shaped spindle at $25 \mathrm{rpm}$ in all cases.

Prior to use, the dissolution media were equilibrated at $37^{\circ} \mathrm{C}$ overnight to deaerate the medium so that bubble formation, due to escape of dissolved gasses during the test, was minimized.

The tests for the diltiazem products were conducted using $900 \mathrm{~mL}$ water. The amount of diltiazem dissolved in each vessel was determined at various time intervals; up to three hours for the IR products and 24 hours for the ER and SR products. The dissolution sampling was achieved using an automated sampling system connected to an online UV diode-spectrophometer (Agilent 8453). The quantitation of 
diltiazem was done by ultraviolet absorbance at $240 \mathrm{~nm}$ of filtered portions of the solutions under test, in comparison with a reference solution having a known concentration of diltiazem standard [5].

Data Analysis: The data were collated and analysed using SAS software (SAS Institute, Cary, NC).

\section{RESULTS}

Fig. (1) shows dissolution results/profiles of three release types of products tested in water, as the dissolution medium, using the crescent-shaped spindle at $25 \mathrm{rpm}$. It is to be noted that all products showed dissolution behaviour as expected, i.e. different types of release characteristics are observed; fast, slow and slower. Therefore, it is evident that a common dissolution test condition may be possible for an appropriate comparison of release characteristics of different product types.

Table 1 summarizes the percent drug release with \%RSD, at plateau, for the individual products. It is evident that using crescent-shaped spindle release of the total $(100 \%)$ of the expected amount of drug, with tight $\% \mathrm{RSD}$, is accounted for in all cases.

\section{DISCUSSION}

It would be prudent that a discussion should first be provided to highlight the superiority of the proposed procedure itself using crescent-shaped spindle in comparison with the use of current apparatuses such as USP Paddle and Basket apparatuses. This will help in leading the description and discussion which makes the proposed method useable for estimating other parameters such as assay and content uniformity $(\mathrm{CU})$ of dosage units.

To consider the superiority of the dissolution apparatus and/or method, it would be advantageous to describe first the desired or required features of an appropriate dissolution test against the current practices and then consider further extension of its use for other tests such as assay and CU of dosage units.

A desired feature of a dissolution test should be that it can estimate potential drug release characteristics of a product in the GI tract environment and do so in a reproducible manner [6]. The human GI tract environment remains the same for all products, therefore, a dissolution test procedure should also be capable of evaluating drug release characteristics of products using similar experimental test condition i.e. procedure should be common or product independent. The testing procedure should also be able to provide drug release characteristics of different products, whether from the same manufacturer with variation in products including multiple strength products, or among products from different manufacturers (which would involve different formulations and manufacturing processes).

Presently employed dissolution testing procedures, in particular using the USP Paddle and Basket, utilize product dependent test procedures [5]. Therefore, not only would results have limited bio-relevancy (reflection of poor GI-tract behaviour), but comparison of drug release characteristics within and between product categories may not be possible. For example, if immediate-release (IR) and extended-release
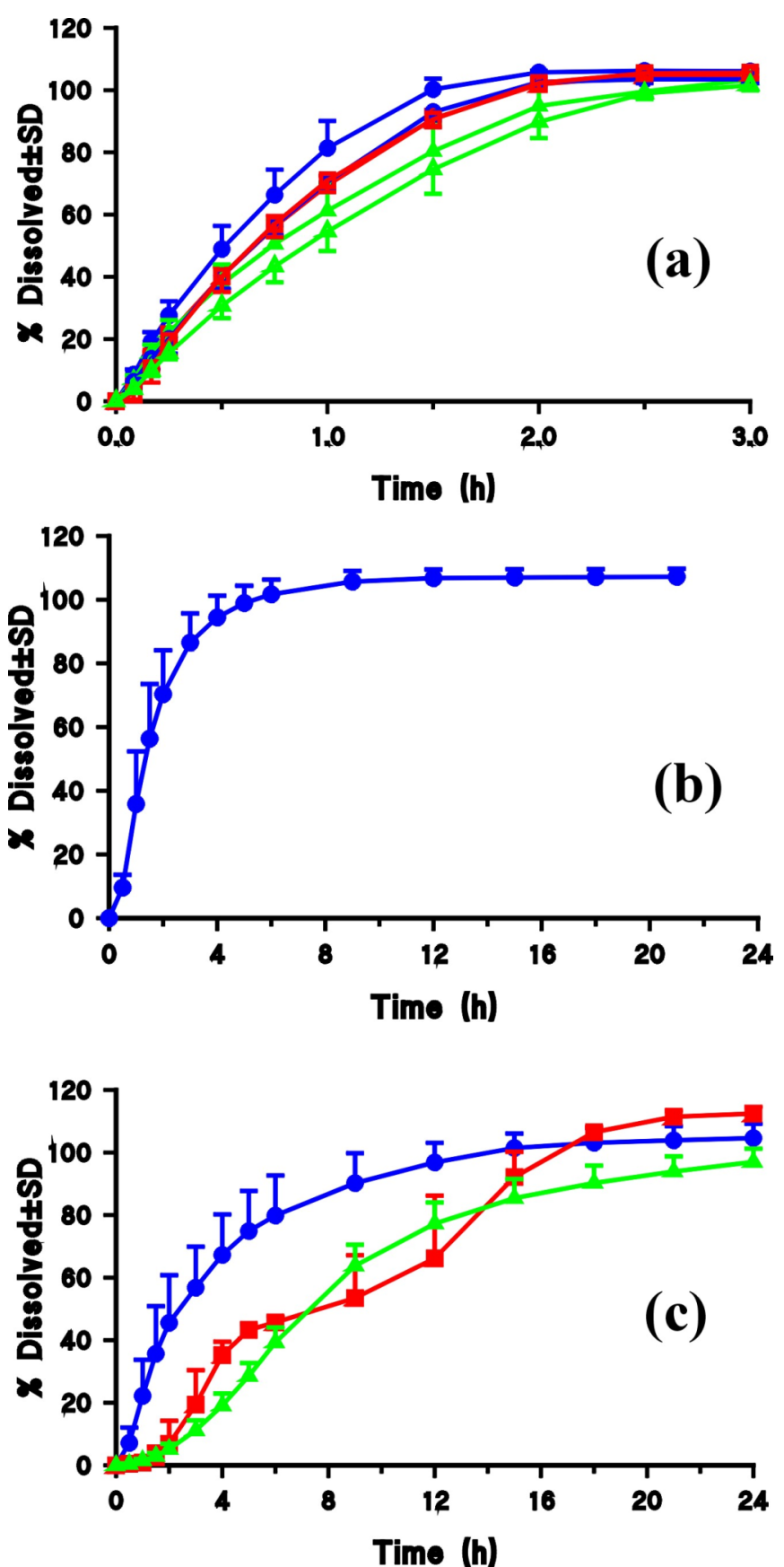

Fig. (1). Drug release profiles of diltiazem products using the crescent-shaped spindle at $25 \mathrm{rpm}$. (a) IR-tablets, solid and open symbols represent $30-$ and $60-\mathrm{mg}$ tablets products respectively, from three manufacturers; (b) ER-Capsules, 120-mg strength of $12 \mathrm{~h}$ dosing capsule products; (c) ER-Capsules (bottom) of 24-dosing, representing products from three manufacturers.

(ER) products are tested by their respective methods, it would not be possible to decide whether drug dissolution characteristics are related to the product or the procedure used.

Recently it has been shown that, by computer simulated models and experimental studies, the commonly used apparatuses (Paddle and Basket) have an artefact because of poor stirring and mixing capability and thus the testing leads to highly variable results [7-9]. 
Table 1. Summary of Percentage Drug Release Values at Plateau Obtained for the Products Evaluated Using Crescent-Shaped Spindle Rotating at $25 \mathrm{rpm}$ Using Water $(900 \mathrm{~mL})$ as Dissolution Medium

\begin{tabular}{|c||c|c|c|c|}
\hline \multicolumn{1}{|c||}{ Product Type } & Manufacturer & Strength (mg) & Mean, \%RSD & Range \\
\hline \hline \multirow{2}{*}{ IR - Tablets (Results at 3 h) } & \multirow{2}{*}{ A } & 30 & $106.1,0.4$ & $105.3-106.7$ \\
\cline { 2 - 5 } & \multirow{2}{*}{ B } & 60 & $103.4,1.2$ & $102.0-104.6$ \\
\cline { 2 - 5 } & & 30 & $104.8,1.0$ & $103.2-106.2$ \\
\cline { 2 - 5 } & \multirow{2}{*}{ C } & 60 & $105.6,1.4$ & $103.9-108.4$ \\
\cline { 2 - 5 } & & 30 & $103.4,1.1$ & $102.0-104.8$ \\
\hline \multirow{2}{*}{ ER - Capsules (12-h dosing) (Results at 12h) } & A & 60 & $106.8,2.6$ & $103.6-111.0$ \\
\hline \multirow{2}{*}{ ER - Capsules (24-h dosing) (Results at 24h) } & A & 120 & $104.6,4.4$ & $99.9-112.8$ \\
\cline { 2 - 5 } & B & 120 & $112.5,1.9$ & $110.3-116.3$ \\
\hline
\end{tabular}

Table 2. Suggested Experimental Procedures for the Evaluation of Dissolution Characteristics of Diltiazem Products According to Respective USP Monographs [5]

\begin{tabular}{|c|c|c|c|c|c|}
\hline Product Type & USP Test \# & Apparatus (rpm) & Medium & Sampling Times & $Q$ at Last Sampling \\
\hline IR - Tablets & & Paddle (75) & Water & $30 \mathrm{~min}$. and $3 \mathrm{~h}$ & NLT $75 \%$ \\
\hline \multirow{4}{*}{ ER - Capsules (12h-dosing) } & 1 & Paddle (100) & Water & 3,9 and $12 \mathrm{~h}$ & NLT $70 \%$ \\
\hline & 4 & Paddle (100) & Water & $4,8,12$, and $24 \mathrm{~h}$ & NLT $80 \%$ \\
\hline & 5 & Paddle (50) & 0.05M Phosphate & 1,3 , and $8 \mathrm{~h}$ & NLT $80 \%$ \\
\hline & 10 & Basket (100) & 0.05 M Phosphate & $1,6,9$ and $24 \mathrm{~h}$ & NLT $80 \%$ \\
\hline \multirow{9}{*}{ ER - Capsules (24h-dosing) } & 2 & Paddle (100) & Water & $1,4,10$ and $15 \mathrm{~h}$ & NLT $80 \%$ \\
\hline & 3 & Paddle (100) & $0.1 \mathrm{~N} \mathrm{HCl}$ & $6,12,18,24$ and $30 \mathrm{~h}$. & NLT $85 \%$ \\
\hline & 6 & Basket (100) & Water & $2,4,8,12$ and $16 \mathrm{~h}$ & NLT $80 \%$ \\
\hline & 7 & Paddle (100) & Acetate Buffer ( $\mathrm{pH} 4.2)$ & $1,4,10$ and $15 \mathrm{~h}$ & NLT $80 \%$ \\
\hline & 8 & Paddle (100) & Water & $1,4,10$ and $15 \mathrm{~h}$ & NLT $80 \%$ \\
\hline & 9 & Paddle (75) & $\begin{array}{l}\text { 1. } 0.1 \mathrm{~N} \mathrm{HCl} \\
\text { 2. Phosphate buffer (pH 7.5) }\end{array}$ & $\begin{array}{c}\text { Medium 1.2h } \\
\text { Medium 2.2,12, 18, } 24 \mathrm{~h}\end{array}$ & NLT $80 \%$ \\
\hline & 11 & Paddle (100) & $0.1 \mathrm{~N} \mathrm{HCl}$ & $1,6,12$, and $18 \mathrm{~h}$ & NLT $85 \%$ \\
\hline & 12 & Paddle (100) & Water & $2,8,14$ and $24 \mathrm{~h}$ & NLT $80 \%$ \\
\hline & 13 & Basket (100) & Water & $2,8,14$ and $24 \mathrm{~h}$ & NLT $80 \%$ \\
\hline
\end{tabular}

NLT $=$ not less than.

To accommodate this artefact, dissolution is evaluated using product specific testing conditions, even with a number of test procedures within a product type (e.g. ER products), usually with wide tolerances. An example of this situation is the USP monographs for the testing of diltiazem products [5], for which there are 13 different official experimental procedures described. A summary of these procedures is provided in Table 2. It should further be noted that these thirteen test conditions and standards are for products of a single drug, and products from other drug products have their corresponding different conditions listed in the compendeial monographs. Therefore, such an approach would not provide an objective and comparative assessment of drug release characteristics, and thus the quality of the test product.

The new approach based on crescent-shaped spindle appears to address the artefact of the currently used apparatuses, and in particular that of the Paddle [10]. The main difference is that the crescent-shaped spindle provides an efficient stirring and mixing environment, and thus improved dissolution, which is lacking in the currently used Paddle and Basket apparatuses. Based on studies evaluating the use of crescent-shaped spindle it has been suggested that a single set of experimental conditions using the crescent-shaped spindle at an rpm of 25 may provide appropriate and bio- 
relevant product evaluation as an alternative to current practices $[2,11]$.

This study provides evidence of such improved testing to simplify a complex pharmacopeial approach such as that for diltiazem products using the crescent-shaped spindle. The products analysed in this study represent a large variation in their biopharmaceutic attributes, including strengths (30-, $60-$ and $120-\mathrm{mg}$ ), dosage forms (tablets and capsules) and release type (IR and ER), as well as being from several sources (3 manufacturers). Fig. (1) shows drug release characteristics all tested products using a single set of experimental conditions which is $900 \mathrm{~mL}$ of water as dissolution medium with crescent-shaped spindle rotated at $25 \mathrm{rpm}$.

Fig. (2) reflects dissolution profiles drawn on the same axes which would provide appropriate comparative dissolution characteristics among products. However, this may not be possible using the current pharmacopeial procedure as products may be analyzed using different procedures.

The bio-relevancy of these results may be concluded from the observation that all products have been tested using a single testing environment as is the case for the single GI tract environment which these products are expected to go through in humans. Each product behaves as would be expected for the release of the drug in humans, i.e. fast (IR), slow (ER 12h) and slower (ER 24h) with corresponding maximum drug release time of 3,12 and $24 \mathrm{~h}$ representing complete drug release in the suggested dosing intervals.

A further limitation of the currently used apparatuses is that because of inefficient product-medium interaction, complete drug release from a product is seldom accounted for. Therefore, to accommodate for this deficiency, tolerances usually are set lower than strength (labelled amount) of the product as shown in Table 2, representing 75 to $85 \%$ of expected drug release. Obviously, using the current dissolution procedures, 15 to $25 \%$ of drug release may not be accounted for even when assay and $\mathrm{CU}$ results would reflect presence of labelled quantity $(100 \%)$ of drug in the product. On the other hand, because of efficient product-medium interaction using the crescent-shaped spindle, complete release of drug, with tight \%RSD, is accounted for in all cases, as illustrated in the Table 1.

Therefore, a dissolution test for diltiazem products using the crescent-shaped spindle not only provides a simpler dissolution test (single vs multiple procedures), but also accounts for total release of the drug from the product.

In short, it may be concluded that the use of crescentshaped spindle not only provides efficient and bio-relevant dissolution testing but may also significantly simplify quality control procedures for dissolution testing unlike those currently described in the pharmacopeias.

The improved stirring and mixing environment obtained using the crescent-shaped spindle provides a more efficient extraction process within a dissolution vessel. Therefore, the new approach not only provides improved dissolution assessment, but may lead to its use in other tests where an efficient extraction step is needed. Two other tests used in quality assessment and which require an extraction step are the assay and CU.

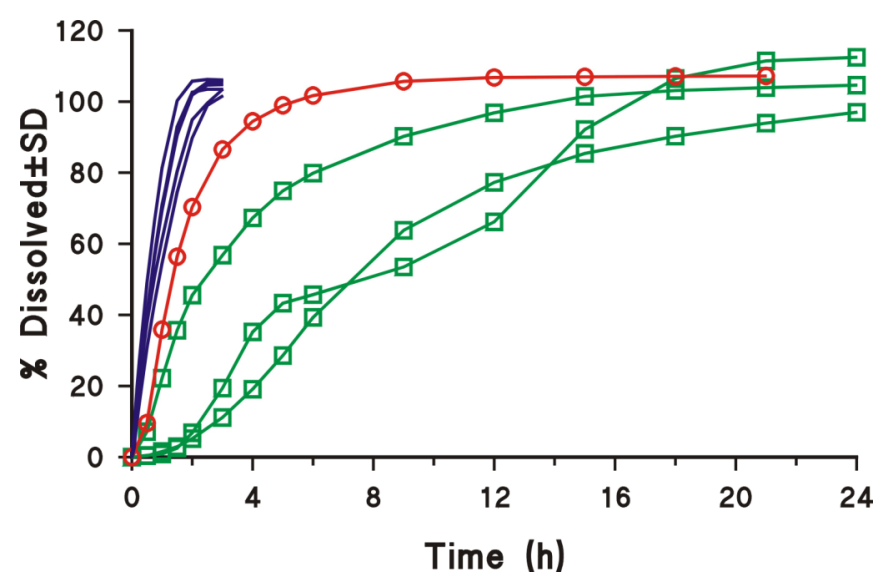

Fig. (2). Drug release profiles of diltiazem products redrawn from Fig. (1) using same time scale (x-axis). IR-products (-), ER-capsules (12h-dosing, - $-\mathrm{O}-$ ) and ER-capsules (24h-dosing, $-\square-)$.

Indeed, if one observes the results at the plateau stage of the dissolution tests in Figs. (1) and (2), these results reflect assay (mean of the dissolution results) and CU (range, with RSD). The values for assay and CU thus obtained are described in Table $\mathbf{1}$ which are similar to those expected in the pharmacopeia.

One may argue that the assay and uniformity of dosage units tests based on the dissolution testing may be more appropriate since the evaluation is conducted using a physiologically relevant environment. As per the USP monograph for diltiazem products [5], the assay is conducted using an extraction step with methanol, while dissolution tests are to be conducted using aqueous-based medium. Therefore, if one employs, the drug dissolution test using the crescentshaped spindle, testing for assay and $\mathrm{CU}$ would conducted under more physiologically relevant conditions.

The described approach/concept for obtaining CU and assay values from dissolution results provides values similar to those one would expect to obtain from the pharmacopeial methods. However, direct (statistical) comparison of values obtained using pharmacopeial methods and the new approach may not be possible since dissolution based testing requires different multiples ( 6 dosage units) than for $\mathrm{CU}$ and assay, where tests are conducted in multiple of 20 or 30. However, if evaluation is done using the dissolution based approach there may not be any need for conducting different statistical analyses, providing further simplification of the procedure and the results will be directly comparable and related to one another.

In short, for appropriate quality control tests, the following four criteria appear to be necessary: (1) for accurate comparative analysis, including for dissolution testing, multiple products should be analysed using a common set of experimental conditions; (2) in the case of dissolution testing maximum drug content should be released within the prescribed dosage interval; (3) the labelled amount of drug should be accounted for in all tests including assay, CU and dissolution; (4) the variability in dissolution results at the plateau should be reflective of uniformity of dosage unit or CU. 
Considering these criteria, current practices may lack the appropriate quality control testing aspect, since multiple dissolution testing conditions are required as reflected in Table 2. The release of complete drug content is usually not accounted for, as reflected by the setting tolerances as Q in the range of 75 to $80 \%$. Further, such lower ranges would reflect higher variabilities in the results, which may not be reflective of product quality. In some cases, test procedures (at least in three for diltiazem procedures) require testing beyond the prescribed dosing interval, e.g. 24 hours for $12 \mathrm{~h}$ products and $30 \mathrm{~h}$ for 24 hour products. Thus, there is a disconnect between the testing time and duration of time the drug is expected to be released.

However, by testing using the crescent-shaped spindle all the above mentioned objectives may easily be achieved. Therefore, it may be concluded that the use of crescentshaped spindle may provide a better alternative to the current practices of quality control testing of pharmaceutical products. A single dissolution procedure using crescent-shaped spindle may be used to establish assay, CU and dissolution results. Obviously, such a simplified approach would result in significant economical benefits.

\section{ACKNOWLEDGEMENT}

The skilful technical help provided by Dr. J. Dapaah is greatly appreciated.

\section{REFERENCES}

[1] Qureshi, S.A.; Shabnam, J. Eur. J. Pharm. Sci., 2003, 55, 291.

[2] Qureshi, S.A. Eur. J. Pharm. Sci., 2004, 23, 271.

[3] Qureshi, S.A. Dissolution Technol., 2004, 11, 13.

[4] USP. The United States Pharmacopeial Convention, Inc. Rockville, MD., 2005, pp. 2412-2422.

[5] USP. Diltiazem Tablets and Extended-Release Capsules monogrphs. - The United States Pharmacopeial Convention, Inc. Rockville, MD., 2005, pp. 655-658.

[6] FDA Guidance document for Industry. Extended Release Oral Dosage Forms: Development, Evaluation, and Application of In $\mathrm{Vi}$ tro/In Vivo Correlations. http://www.fda.gov/cder/guidance/ 1306fnl.pdf.

[7] McCarthy, L. G.; Kosiol, C.; Healy, A.M.; Bradley, G.; Sexton, J.C.; Corngan, O.L. AAPS PharmSciTech, 2003, 4, 172.

[8] Muzzio, F.J.; Kukura, J.; Baxter, J.L. Int. J. Pharm., 2005, $292,17$.

[9] Qureshi, S.A.; Shabnam, J. Eur. J. Pharm. Sci., 2001, 12, 271.

[10] Qureshi, S.A. J. Pharm. Pharmacol., 2004, 56, 1135.

[11] Spagnoli, S.; Di Maso, M. Dissolution Technol., 2006, 13, 20. 